\title{
CD36 - a plausible modifier of disease phenotype in familial adenomatous polyposis
}

\author{
Merran Holmes ${ }^{1,2 \dagger}$, Toni Connor ${ }^{1,3 \dagger}$, Christopher Oldmeadow ${ }^{4}$, Peter G. Pockney ${ }^{1,2}$, Rodney J. Scott ${ }^{3,5}$ \\ and Bente A. Talseth-Palmer ${\text {, }, 6,7^{*}}_{\text {iD }}$
}

\begin{abstract}
Background: Familial adenomatous polyposis (FAP) is a well characterised genetic predisposition to early onset colorectal cancer (CRC) that is characterised by polyposis of the colon and rectum. Animal models have consistently suggested the role of modifier genes in determining disease phenotype, yet none have been substantiated in the human population. The mouse homologue of cluster of differentiation 36 (CD36) has been proposed as a modifier of disease in the MIN mouse model of FAP.

Methods: Three single nucleotide polymorphisms (SNPs); rs1049673, rs1761667 and rs1984112 in CD36, have been investigated in 275 FAP patients to determine if they were associated with age of polyposis or risk of developing disease.

Results: The results revealed a substantially lower age of polyposis diagnosis for patients belonging to the severe FAP group (harbouring adenomatous polyposis coli (APC) variants in the mutation cluster region (MCR)) and high age for patients in the attenuated familial adenomatous polyposis (AFAP) group for SNPs rs1761667 and rs1984112.

Conclusions: This study provides evidence for patients belonging to the MCR and AFAP groups harbouring specific genotypes for SNPs in CD36 to initiate screening/treatment for FAP at much earlier (MCR) and much later (AFAP) ages than the norm in today's clinical practice. The findings need to be verified in an independent FAP patient cohort.
\end{abstract}

Keywords: FAP, Disease phenotype, Polyposis, Modifier gene, CD36

\section{Background}

Familial adenomatous polyposis (FAP) is a rare autosomal dominantly inherited cancer syndrome that predisposes to colorectal cancer at unusually young ages, with disease penetrance that approaches 100\% [1]. The diagnosis of FAP is based on the presence of hundreds to thousands of colorectal adenomatous polyps that appear in the colon and rectum during the first and second

\footnotetext{
* Correspondence: Bente.Talseth-Palmer@newcastle.edu.au

${ }^{+}$Merran Holmes and Toni Connor contributed equally to this work.

${ }^{5}$ School of Biomedical Science and Pharmacy, Faculty of Health and Medicine, University of Newcastle and Hunter Medical Research Institute, Newcastle, Australia

${ }^{6}$ Department of Clinical and Molecular Medicine, Faculty of Medicine and Health Sciences, Norwegian University of Science and Technology,

Trondheim, Norway

Full list of author information is available at the end of the article
}

decades of life [2]. The disease is typically characterised by a carpeting of polyps within the colon and rectum and without appropriate prophylactic treatment approximately $95 \%$ of patients will develop CRC by the age of 40 years $[2,3]$.

FAP has been shown to be a result of germline mutations occurring in the tumour suppressor gene adenomatous polyposis coli (APC), which is located on chromosome 5q21-22.2 and is a key regulator of the Wnt signalling pathway [4]. Genotype/phenotype correlations are well described in FAP and are associated with variants that inactivate $A P C$ [5]. Genotype/phenotype studies reveal essentially two subgroups of FAP, one that predisposes to a milder form of disease known as attenuated FAP (AFAP) and the other more common form of the disease that is associated with the more severe

(C) The Author(s). 2018 Open Access This article is distributed under the terms of the Creative Commons Attribution 4.0 International License (http://creativecommons.org/licenses/by/4.0/), which permits unrestricted use, distribution, and 
phenotype [6, 7]. Pathogenic variants tend to occur within a mutation cluster region (MCR), bordered by codons 1286 and 1513, encompassing one specific variant (codon 1309) that is associated with the most severe phenotype [8]. Many reports have confirmed the severity of codon 1309 variants but not all patients with this mutation present with similar disease characteristics, with some patients displaying extremely severe polyposis whereas others have significantly less dense polyposis. These evidence suggests that there are other factors (genetic and/or environmental) influencing disease expression [5, 9].

Several studies have focused on the identification of candidate genes in mouse models of FAP (known as MOM - modifiers of MIN), reviewed in and recently 7 potential modifier genes (CD36 being one of them) have been identified that affect tumour multiplicity in this model [10]. The mouse homologue of CD36 has been proposed as a modifier of disease in the MIN mouse model of FAP [10]. CD36, a class B scavenger, located on chromosome 7q11.2, encodes a membrane glycoprotein found on epithelial cells of the breast, kidney and gut $[11,12]$ and has been shown to be involved in angiogenesis, atherosclerosis, inflammation, lipid metabolism and phagocytic clearance of apoptotic cells. There are several reports suggesting that CD36 may play a role in affecting different disease phenotypes that include Alzheimer's disease, heart disease, cancer, obesity, type 2 diabetes mellitus (T2DM) and associated metabolic disease [13-15].

Due to the modifier role of CD36 in the mouse and its association with malignancy the aim of the current study was to determine whether three single nucleotide polymorphisms (SNPs) occurring in CD36 were associated with modifying disease severity in a large cohort of molecularly diagnosed FAP patients.

\section{Methods}

The patient cohort consisted of 275 individuals who carry a pathogenic germline mutation in $A P C$ (population I), belonging to 137 families. The patients were ascertained between 1997 and 2016, and analysed at Pathology North, Newcastle, NSW, Australia. From the 275 patients, 61 were diagnosed with AFAP due to their causative variant location, 34 had mutations in the mutation cluster region (MCR) and the remainder 179 was recognised as classic FAP. Mutation groups were divided according to polyposis phenotype; severe (APC MCR = codons 1250-1513), attenuated (APC AFAP $=5$ 'end spanning exon 3 to 5, 3'distal end and those in exon 9) and intermediate (APC $=$ the rest of the gene)). Population II $(n=214)$ excluded AFAP (attenuated) patients to see whether that influenced the results for the SNPs.

Several patients remained asymptomatic at the time of referral as they were too young to present with disease.
The clinical data collected for this study included age of diagnosis of polyposis and age at last follow-up for patients undiagnosed with polyposis.

The 275 FAP patient samples were used for genotyping of SNPs in CD36; rs1049673 (C> G), rs1761667 $(\mathrm{G}>\mathrm{A})$ and rs1984112 (A > G) on chromosome 7q21.11 using TaqMan SNP assays (Applied Biosystems). SNP rs1049673 is located in exon 15 (3'-UTR), while the two others are intronic variants flanking exon 1A [16]. SNP rs1761667 has been shown to reduce protein expression, while 3'-UTR variants often contain regulatory regions that post-translationally influence gene expression [17]. The 3 SNPs are in strong LD with three haplotype blocks described by the HapMap database [18]. Thermo-cycling was undertaken according to the TaqMan SNP Genotyping Assay Protocol, involving; $10 \mathrm{~min}$ at 95 degrees; 40 cycles of $15 \mathrm{~s}$ at 95 degrees; and $1 \mathrm{~min}$ at 60 degrees. Results were read using the 7500 standard real-time PCR system (Applied Biosystems). Raw data was analysed using TaqMan Genotyper Software (Life Sciences, Foster City, CA).

A Pearson's Chi-square test was used to evaluate deviation from the expected Hardy-Weinberg equilibrium (HWE). Statistical analysis was performed using Stata 12.1 (StataCorp LP, TX USA). We applied Bonferroni correction for multiple testing, resulting in a corrected significance threshold of $p=0.0167$ ( 0.05 divided by the 3 SNPs tested). Due to the nature of the disease and prophylactic surgery, diagnosis of polyposis was used as endpoint for analysis. Variation in age of polyposis diagnosis between each SNP; wildtype genotype (homozygous for wildtype allele), heterozygote and variant genotype (homozygous for variant allele) and mutation group (based on mutation location as described above); was examined using Kaplan-Meier plots. Individuals free from polyposis were censored at their age at last follow up. Wilcoxon's (Breslow), Log-rank and Tarone-Ware tests were used to examine homogeneity of the Kaplan-Meier plots. The log-rank test is more sensitive to differences later in time due to equal weighting over the curve, where the Wilcoxon weights the early differences higher than the later differences using the number at risk in the weighting. The Tarone-ware test uses the square root of the number at risk in the weighting. All three tests were required to be significant for results to be considered reliable. Cox regression models were used to provide a formal Wald-test of interaction (global interaction test) between APC mutation groups and SNPs genotypes, taking into account family ID as a group variable.

\section{Results}

Of the 275 patients belonging to 137 families, 28 patients had a diagnosis of CRC and 182 a diagnosis of polyposis (population I). Of these there were 152 females and 123 males. The average age of polyposis was 36 years 
and for CRC 45 years. Of the 275 patients, 61 were classified as AFAP according to APC mutation location and the cohort was also analysed without AFAP patients (population II).

Patient samples that failed genotyping (24 for rs1049673, 13 for rs1761667 and 28 for rs1984112) were excluded from further analysis for the SNP in question. None of the SNPs deviated from HWE.

In the sample cohort we observed a statistically significant difference between the phenotypic FAP groups (based on $A P C$ variant location) and age of polyposis diagnosis $(\log$-rank $p=0.0010$, Wilcoxon $p=0.0004$ and Tarone ware $p=0.0005$ ); age where $50 \%$ of population was polyposis free was 46 years for APC AFAP, 29 years for APC MCR and 37 years for APC, see Fig. 1. There was no statistically significant difference between males and females regarding age of diagnosis of polyposis in this cohort (see Fig. 2). Tests for equality of survival distributions between genotypes of the CD36 SNPs rs1049673, rs1781667 and rs1984112 for population I was not statistically significant, shown in Table 1 and Fig. 2. Similar results were obtained for population II (data not shown).

To determine whether there was a differential effect of CD36 genotypes depending on APC mutation location (mutation group) on age of diagnosis of polyposis we stratified the Kaplan-Meier curves into the three possible phenotypic groups (APC, APC MCR and APC AFAP), see Table 2. For two of the SNPs, rs1761667 and rs1984112, a trend (approaching statistical significance) shows that individuals harbouring the homozygous

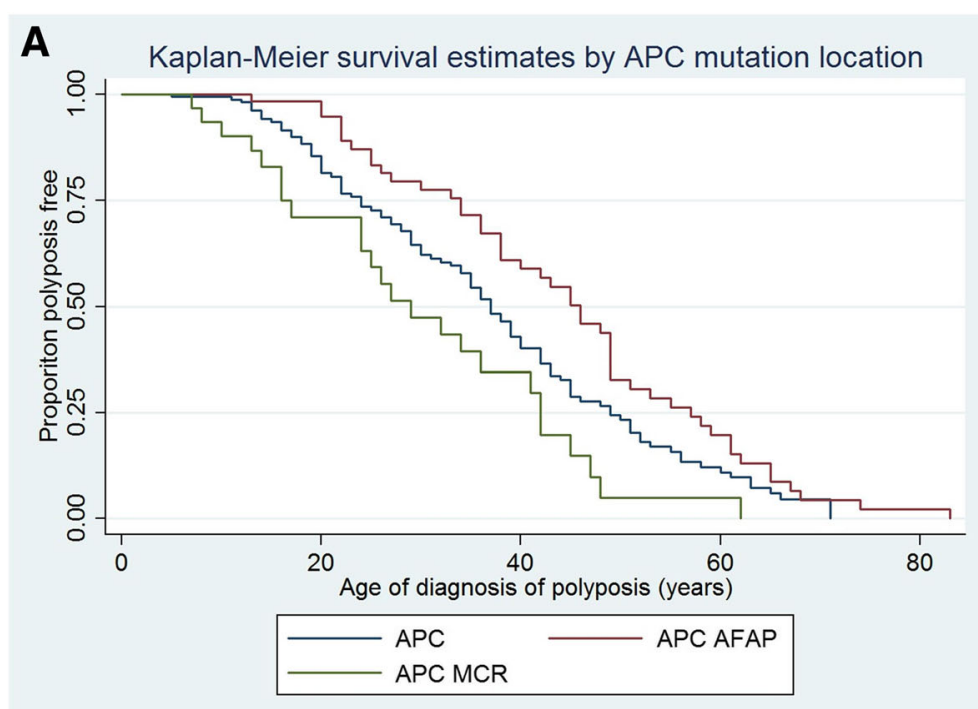

B

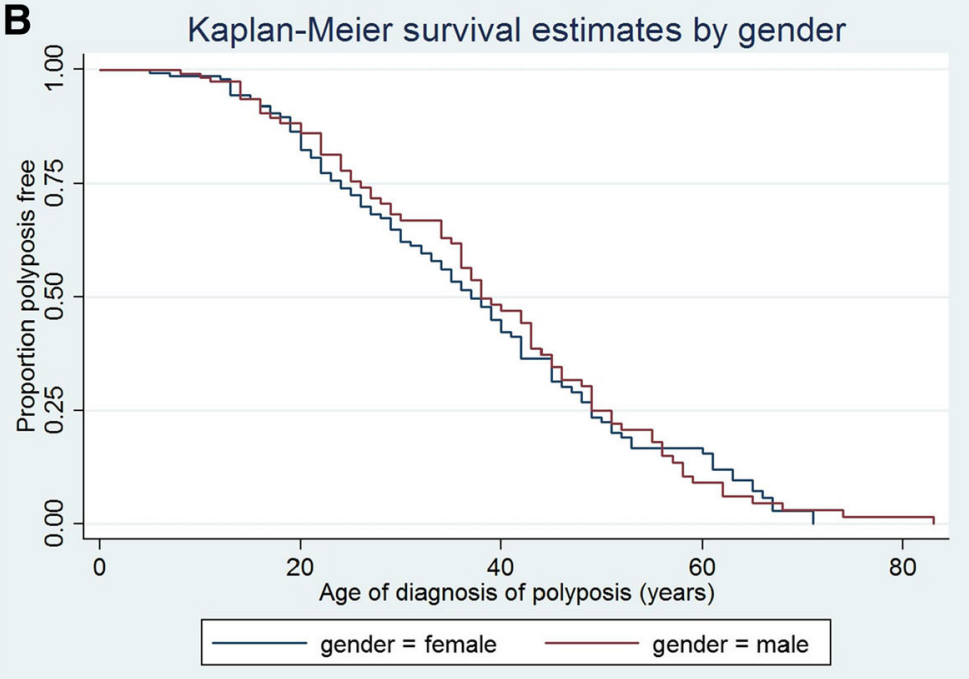

Fig. 1 Shows the difference in age of diagnosis of polyposis between a) mutation-groups divided by APC mutation location $(n=275)$. APC $=$ classic FAP APC MCR = mutation cluster region (more severe phenotype) and APC AFAP (attenuated phenotype) - statistically significant; Log-rank $p=0.0010$, Wilcoxon $p=0.0004$ and Tarone ware $p=0.0005$, and $\mathbf{b})$ between female and males $(n=275)$. Not statistically different 


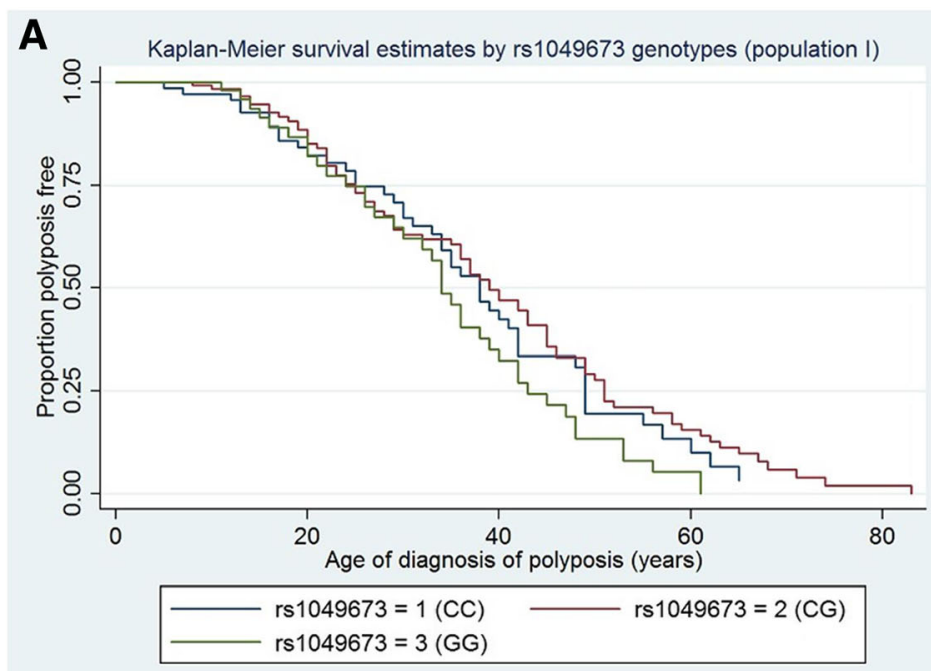

B Kaplan-Meier survival estimates by rs1761667 genotypes (population I)

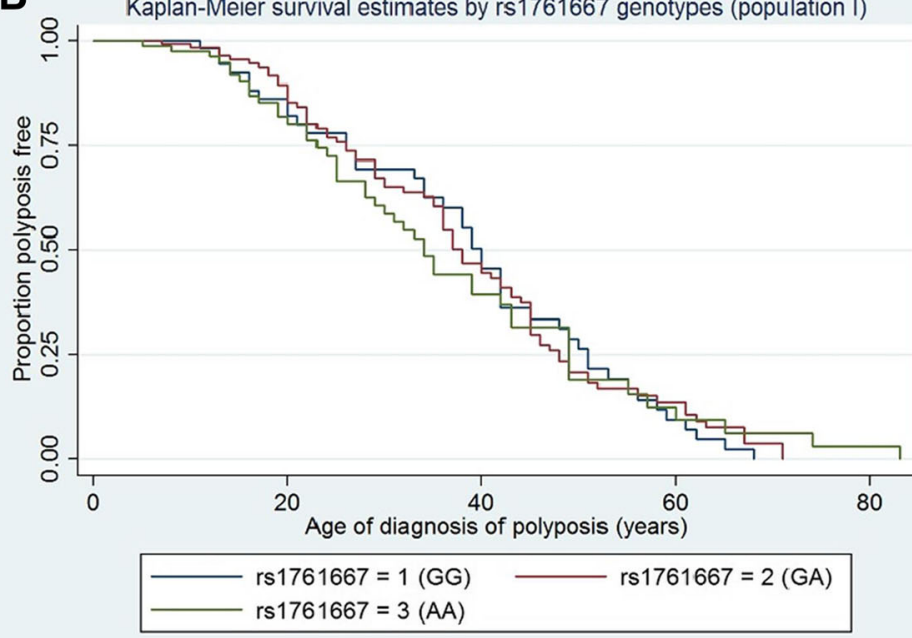

C Kaplan-Meier survival estimates by rs 1984112 genotypes (population I)

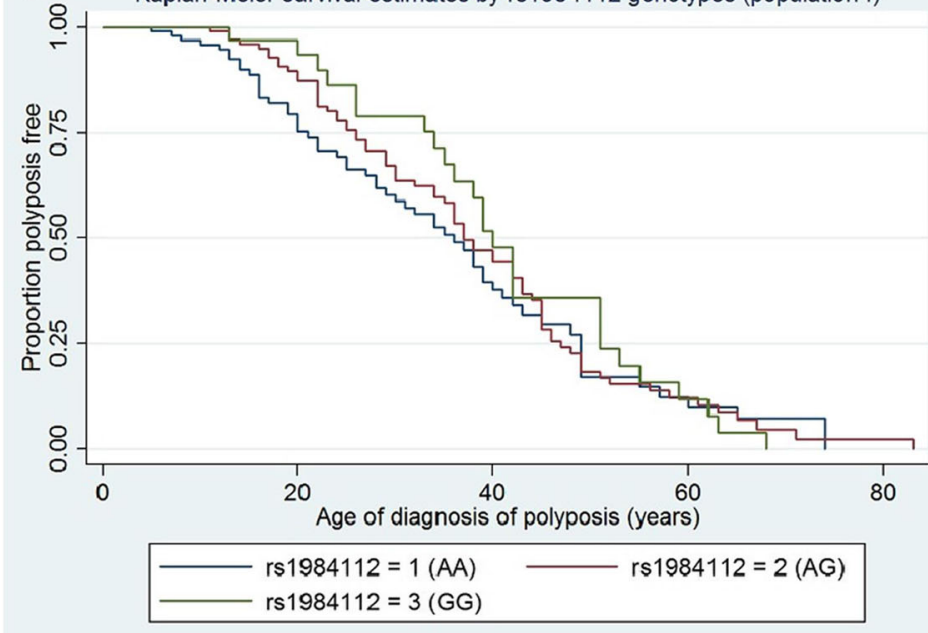

Fig. 2 Kaplan-Meier curves for SNP a) rs1049676, b) rs1761667 and c) rs1984112 stratified by genotypes 
Table 1 Population I (FAP and AFAP): The table lists genotypes for each SNP, number of polyposis cases and age where 50\% of population I is polyposis free for each genotype

\begin{tabular}{|c|c|c|c|c|c|c|c|}
\hline \multirow[t]{2}{*}{ SNP } & \multicolumn{3}{|c|}{ Genotypes $^{\mathrm{a}}(\mathrm{n})$ (polyposis cases (n)/age at 50\%) } & \multirow[b]{2}{*}{ Total n (polyposis cases) } & \multicolumn{3}{|l|}{$p$-values } \\
\hline & 1 & 2 & 3 & & Log-Rank & Tarone-ware & Wilcoxon \\
\hline rs1049673 & $74(45 / 38)$ & $124(81 / 39)$ & $53(39 / 34)$ & $251(165)$ & 0.0765 & 0.2210 & 0.4075 \\
\hline rs1761667 & $56(44 / 40)$ & $121(82 / 38)$ & $85(46 / 34)$ & $262(172)$ & 0.8891 & 0.5861 & 0.4527 \\
\hline rs1984112 & $102(57 / 36)$ & $108(76 / 37)$ & $37(26 / 40)$ & 247 (159) & 0.6108 & 0.2215 & 0.0978 \\
\hline
\end{tabular}

aenotypes: 1 = wildtype, 2 = heterozygous and 3 = variant

variant genotype and homozygous wildtype genotype have a remarkably lower age of polyposis diagnosis in patients with causative variants residing in the MCR (APC MCR), even though not statistically significant (see global interaction test in Table 2). Patients belonging to the APC MCR group, with the variant genotype (AA) for SNP rs1761667 and wildtype genotype (GG) for SNP rs1984112 (independent of each other) developed polyposis on average at 16 years of age, which is 13 years younger than the average age for this mutations group in the current sample cohort. Equally interesting is the high age of polyposis diagnosis (58 years) in patients belonging to the APC AFAP group harbouring the wildtype genotype (GG) for SNP rs1761667.

\section{Discussion}

The disease spectrum observed in patients with germline causative variants in $A P C$ has been associated with a genotype/phenotype correlation where some variants are linked to severe and others milder forms of the disease [9]. Notwithstanding, phenotype differences in patients with the same mutation are still observed and modifiers of disease severity still await discovery. This study was undertaken to determine whether CD36 variants could account for phenotypic variation observed in FAP patients, and we have observed a remarkable low age of polyposis diagnosis (16 years) in patients with $A P C$ mutations in the MCR harbouring the variant (AA) genotype of rs1761667 or the wildtype (AA) genotype of rs1984112, independent of each other and a much higher age of polyposis diagnosis for patients harbouring the wildtype genotype (GG) of rs1761667 in the APC AFAP mutation group. This is 13 year younger and 12 years older than the average age of polyposis in this sample cohort for patients with mutations in the MCR and AFAP region, which were 29 and 46 years of age respectively, before taking into account the CD36 SNP genotypes.

FAP has long been categorised as a condition that affects younger people from their early teenage years. Virtually all patients will have developed polyposis a decade after the appearance of their first adenomas and, if left untreated, CRC by 40 years of age [1, 19]. But the phenomenon of anticipation has been described and age of disease onset decreases in successive generations [20]. Similar to the published penetrance estimates [6-8], the average age of polyposis and CRC diagnosis in our FAP population were 36 and 45 years of age, respectively, indicating that our sample cohort is consistent with other FAP populations. Our sample cohort clearly reveals a significant difference in disease expression among the three different phenotypic FAP groups; severe (APC MCR), intermediate (APC) and attenuated (APC AFAP) FAP, indicating that those individuals harbouring $A P C$ mutations in the MCR, should initiate screening/treatment at much earlier ages than other FAP or AFAP patients. But on top of this, the evidence presented herein suggest that patients belonging to the severe FAP group (APC MCR) who harbour the variant genotype for rs1761667 or wildtype genotype for rs1984112, should commence screening for polyposis at younger ages than their counterparts who do not carry these modifying alleles. Also interesting from a surgical perspective is that

Table 2 Population I (FAP and AFAP): The table lists combination of genotypes ( 1 = wildtype genotype, $2=$ heterozygote genotype and 3 = variant genotype) and phenotypic FAP group ( 1 = APC, $2=$ APC MCR and $3=$ APC AFAP) for each SNP and age where $50 \%$ of population I is polyposis free for each genotype. The mutation group/SNP interaction is the $p$-value from a joint test of the interaction parameters of a Cox model

\begin{tabular}{|c|c|c|c|c|c|c|c|c|c|c|c|c|c|}
\hline \multirow{3}{*}{$\begin{array}{l}\text { SNP } \\
\text { genotype }\end{array}$} & \multicolumn{9}{|c|}{ Age where $50 \%$ of population is polyposis free for each genotype ${ }^{a}$} & \multirow{2}{*}{\multicolumn{3}{|c|}{$\begin{array}{l}\text { Test equality of survivor functions } \\
p \text {-values }\end{array}$}} & \multirow{3}{*}{$\begin{array}{l}\text { Cox model Mutationgroup/ } \\
\text { SNP interaction } \\
p \text {-values Wald-test }\end{array}$} \\
\hline & \multicolumn{3}{|c|}{$\overline{A P C}$ (mutation group 1) } & \multicolumn{3}{|c|}{$\begin{array}{l}\text { APC MCR } \\
\text { (mutation group 2) }\end{array}$} & \multicolumn{3}{|c|}{$\begin{array}{l}\text { APC AFAP } \\
\text { (mutation group 3) }\end{array}$} & & & & \\
\hline & 1 & 2 & 3 & 1 & 2 & 3 & 1 & 2 & 3 & Log-Rank & Tarone-ware & Wilcoxon & \\
\hline rs1049673 & 38 & 37 & 29 & 29 & 25 & 36 & 49 & 46 & 38 & 0.0082 & 0.0062 & 0.0057 & 0.3090 \\
\hline rs1761667 & 39 & 37 & 34 & 36 & 41 & 16 & 58 & 38 & 49 & 0.0010 & 0.0011 & 0.0015 & 0.0663 \\
\hline rs1984112 & 35 & 37 & 39 & 16 & 32 & 42 & 49 & 42 & 51 & 0.0036 & 0.0007 & 0.0001 & 0.4608 \\
\hline
\end{tabular}

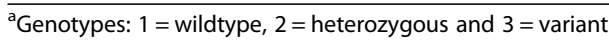


patients in the lower risk groups; especially AFAP patients harbouring the wildtype genotype for rs1761667 could be screened for longer before requiring prophylactic surgery.

CD36 has been identified as a potentially interesting Mom5 candidate [10] and the only gene found to be differentially expressed in the intestine in the mice used to reveal this modifier (B6 and 12P2 strains). CD36 encodes a scavenger receptor involved in fatty acid processing in the intestine [21]. CD36 has also been associated with increased pro-inflammatory signalling and the production of reactive oxygen species [22], both known to influence tumour development. Previously, decreased expression of CD36 has been observed in breast cancer and it has also been associated with worse prognosis in CRC patients [23, 24], lending weight to its role in tumour development.

For SNP rs1761667 the presence of the A (minor/variant) allele has been associated with decreased fat/sugar intake in obese children and increased BMI in T2DM [25]. Intriguingly, the AA genotype has also been associated with lower BMI [26] and high threshold of gustatory fat detection in obese women [27]. Overall, obesity has been unequivocally associated with an increased risk of colorectal cancer outside of the context of FAP. We were unable to obtain BMI information about our cohort of FAP patients at the time of polyposis development and are therefore unable to correlate these findings with an earlier age of disease onset.

Some limitations of our study should be considered. Due to the standard procedure of colectomy it is almost impossible to obtain accurate information about the age of cancer diagnosis in this population and as such we are unable to determine whether $C D 36$ variants are associated with cancer risk rather than the appearance of polyposis. The number of patients in this study remains limited and to prove beyond doubt the relationship between CD36 and disease development a larger number of FAP samples need to be tested. If these results can be verified in an independent cohort of FAP patients additional clinical information will be required to determine the precise role of CD36 in ameliorating or promoting polyposis development. At present it remains to be revealed as to how CD36 could influence adenoma development, one possible mechanism is its role as an endogenous inhibitor of angiogenesis. Lower levels of CD36 predispose to higher levels of angiogenesis which is required for tumour growth ${ }^{23}$, which potentially explains the earlier age of disease onset in patients with variants that reduce promoter activity (i.e the rare allele of rs1761667 and common allele associated with rs1984112).

\section{Conclusions}

If the findings from this study can be independently verified they will have important consequences with respect to the timing of prophylactic surgery for FAP patients and the overall quality of life for patients who have to undergo such a major life-changing event.

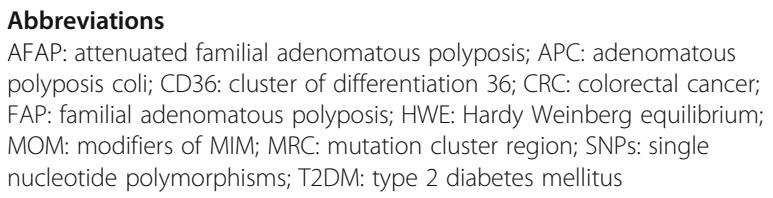

\section{Acknowledgements}

We would like to thank Ashish K. Singh for help with statistical analysis.

\section{Availability of data and material}

All data generated or analysed during this study are included in this published article.

\section{Funding}

This work was supported by the Liaison Committee between the Central Norway Regional Health Authority (RHA) and the Norwegian University of Science and Technology (NTNU), Norway; Møre og Romsdal Hospital Trust, Norway; and the Cancer Institute NSW, Australia.

\section{Authors' contributions}

$\mathrm{MH}$ and TC carried out the molecular genetic studies, OC provided statistical analysis guidance, PGP participated in the study design, RJS and BTP participated in the design of the study and coordination, performed statistical analysis, interpreted and drafted the manuscript. All authors read and approved the final manuscript.

\section{Ethics approval and consent to participate}

All patients consented for the use of their de-identified DNA to be used in future research into their condition. The study was approved by the Hunter New England Human Research Ethics Committee and Human Research Ethics Committee of the University of Newcastle, Newcastle, Australia.

\section{Consent for publication}

Not applicable.

\section{Competing interests}

The authors declare that they have no competing interests.

\section{Publisher's Note}

Springer Nature remains neutral with regard to jurisdictional claims in published maps and institutional affiliations.

\section{Author details}

${ }^{1}$ School of Medicine and Public Health, Faculty of Health and Medicine, University of Newcastle, Newcastle, NSW, Australia. 'Department of Surgery, John Hunter Hospital, Newcastle, Australia. ${ }^{3}$ Pathology North, NSW Health Pathology, Newcastle, Australia. ${ }^{4}$ Centre for Clinical Epidemiology and Biostatistics, University of Newcastle, Newcastle, NSW, Australia. ${ }^{5}$ School of Biomedical Science and Pharmacy, Faculty of Health and Medicine, University of Newcastle and Hunter Medical Research Institute, Newcastle, Australia. ${ }^{6}$ Department of Clinical and Molecular Medicine, Faculty of Medicine and Health Sciences, Norwegian University of Science and Technology, Trondheim, Norway. ${ }^{7}$ Clinic for Medicine, Møre og Romsdal Hospital Trust, Molde, Norway.

Received: 1 March 2018 Accepted: 19 July 2018

Published online: 28 July 2018

References

1. Jass JR. Colorectal polyposes: from phenotype to diagnosis. Pathol Res Pract. 2008;204(7):431-47. https://doi.org/10.1016/j.prp.2008.03.008.

2. Jasperson KW, Burt RW. APC-associated polyposis conditions. 1993.

3. Pagon RAAMP, Ardinger HH, et al. APC-Associated Polyposis Conditions. GeneReviews. 2014:38.

4. Fearnhead NS, Britton MP, Bodmer WF. The ABC of APC. Hum Mol Genet. 2001;10(7):721-33. 
5. Crabtree MD, Tomlinson IPM, Hodgson SV, Neale K, Phillips RKS, Houlston RS. Explaining variation in familial adenomatous polyposis: relationship between genotype and phenotype and evidence for modifier genes. Gut. 2002;51(3):420-3.

6. APC. APC Gene [database on the Internet];2016. Available from: http:// omim.org/entry/611731. Accessed 12 May 2016.

7. van der Luijt RB, Meera Khan P, Vasen HF, Breukel C, Tops CM, Scott RJ, et al. Germline mutations in the 3' part of APC exon 15 do not result in truncated proteins and are associated with attenuated adenomatous polyposis coli. Hum Genet. 1996;98(6):727-34.

8. Nilbert M, Kristoffersson U, Ericsson M, Johannsson O, Rambech E, Mangell P. Broad phenotypic spectrum in familial adenomatous polyposis; from early onset and severe phenotypes to late onset of attenuated polyposis with the first manifestation at age 72. BMC Med Genet. 2008;9:101-. doi:https:// doi.org/10.1186/1471-2350-9-101.

9. Talseth-Palmer BA. The genetic basis of colonic adenomatous polyposis syndromes. Hereditary Cancer Clin Pract. 2017;15(1):5. https://doi.org/10. 1186/s13053-017-0065-X.

10. Otterpohl KL, Gould KA. Genetic dissection of the Mom5 modifier locus and evaluation of Mom5 candidate genes. Mamm Genome. 2015;26(5-6):23547. https://doi.org/10.1007/s00335-015-9567-x.

11. Silverstein RL, Febbraio M. CD36, a scavenger receptor involved in immunity, metabolism, angiogenesis, and behavior. Sci Signal. 2009;2(72): re3. https://doi.org/10.1126/scisignal.272re3.

12. Febbraio $M$, Hajjar DP, Silverstein RL. CD36: a class B scavenger receptor involved in angiogenesis, atherosclerosis, inflammation, and lipid metabolism. J Clin Invest. 2001;108(6):785-91. https://doi.org/10.1172/JCl14006.

13. Love-Gregory L, Sherva R, Sun L, Wasson J, Schappe T, Doria A, et al. Variants in the CD36 gene associate with the metabolic syndrome and high-density lipoprotein cholesterol. Hum Mol Genet. 2008;17(11):1695-704. https://doi.org/10.1093/hmg/ddn060.

14. Sery O, Janoutova J, Ewerlingova L, Halova A, Lochman J, Janout V, et al. CD36 gene polymorphism is associated with Alzheimer's disease. Biochimie. 2017;135:46-53. https://doi.org/10.1016/j.biochi.2017.01.009.

15. Gautam S, Agrawal CG, Banerjee M. CD36 gene variants in early prediction of type 2 diabetes mellitus. Genet Test Mol Biomarkers. 2015;19(3):144-9. https://doi.org/10.1089/gtmb.2014.0265.

16. Ma X, Bacci S, Mlynarski W, Gottardo L, Soccio T, Menzaghi C, et al. A common haplotype at the CD36 locus is associated with high free fatty acid levels and increased cardiovascular risk in Caucasians. Hum Mol Genet. 2004;13(19):2197-205. https://doi.org/10.1093/hmg/ddh233.

17. Love-Gregory L, Sherva R, Schappe T, Qi JS, McCrea J, Klein S, et al. Common CD36 SNPs reduce protein expression and may contribute to a protective atherogenic profile. Hum Mol Genet. 2011;20(1):193-201. https:// doi.org/10.1093/hmg/ddq449.

18. Bokor S, Legry V, Meirhaeghe A, Ruiz JR, Mauro B, Widhalm K, et al. Singlenucleotide polymorphism of CD36 locus and obesity in European adolescents. Obesity (Silver Spring). 2010;18(7):1398-403. https://doi.org/10. 1038/oby.2009.412.

19. Half E, Bercovich D, Rozen P. Familial adenomatous polyposis. Orphanet J Rare Dis. 2009;4:22. https://doi.org/10.1186/1750-1172-4-22

20. Iwama T, Utsunomiya J. Anticipation phenomenon in familial adenomatous polyposis:an analysis of its origin. World J Gastroenterol. 2000;6(3):335-8.

21. Aitman TJ, Glazier AM, Wallace CA, Cooper LD, Norsworthy PJ, Wahid FN, et al. Identification of $\mathrm{Cd} 36$ (fat) as an insulin-resistance gene causing defective fatty acid and glucose metabolism in hypertensive rats. Nat Genet. 1999; 21(1):76-83. https://doi.org/10.1038/5013.

22. Martin-Fuentes P, Civeira F, Recalde D, Garcia-Otin AL, Jarauta E, Marzo I, et al. Individual variation of scavenger receptor expression in human macrophages with oxidized low-density lipoprotein is associated with a differential inflammatory response. J Immunol. 2007;179(5):3242-8.

23. DeFilippis RA, Chang H, Dumont N, Rabban JT, Chen YY, Fontenay GV, et al. CD36 repression activates a multicellular stromal program shared by high mammographic density and tumor tissues. Cancer Discov. 2012;2(9):826-39. https://doi.org/10.1158/2159-8290.CD-12-0107.

24. Tsuchida T, Kijima H, Tokunaga T, Oshika Y, Hatanaka H, Fukushima Y, et al. Expression of the thrombospondin 1 receptor CD36 is correlated with decreased stromal vascularisation in colon cancer. Int J Oncol. 1999;14(1):47-51.

25. Pioltine MB, de Melo ME, Santos A, Machado AD, Fernandes AE, Fujiwara CT, et al. Genetic variation in CD36 is associated with decreased fat and sugar intake in obese children and adolescents. J Nutrigenet Nutrigenomics. 2016; 9(5-6):300-5. https://doi.org/10.1159/000455915.

26. Solakivi T, Kunnas T, Nikkari ST. Contribution of fatty acid transporter (CD36) genetic variant rs1761667 to body mass index, the TAMRISK study. Scand J Clin Lab Invest. 2015;75(3):254-8. https://doi.org/10.3109/00365513.2014.1003596.

27. Mrizak I, Sery O, Plesnik J, Arfa A, Fekih M, Bouslema A, et al. The a allele of cluster of differentiation 36 (CD36) SNP 1761667 associates with decreased lipid taste perception in obese Tunisian women. Br J Nutr. 2015;113(8): 1330-7. https://doi.org/10.1017/S0007114515000343.

\section{Ready to submit your research? Choose BMC and benefit from:}

- fast, convenient online submission

- thorough peer review by experienced researchers in your field

- rapid publication on acceptance

- support for research data, including large and complex data types

- gold Open Access which fosters wider collaboration and increased citations

- maximum visibility for your research: over $100 \mathrm{M}$ website views per year

At BMC, research is always in progress.

Learn more biomedcentral.com/submissions 